Article

\title{
Improving the Dipping Step in Czochraski Process Using Haar-Cascade Algorithm
}

\author{
Le Tran Huu Phuc ${ }^{\circledR}$, HyeJun Jeon, Nguyen Tam Nguyen Truong * and Jung Jae Hak* \\ Chemical Engineering Department, Yeungnam University, Gyeongsan-Si/38541, Korea; \\ phucleth@ynu.ac.kr (L.T.H.P.); daivjeon@ynu.ac.kr (H.J.) \\ * Correspondence: tamnguyentn@ynu.ac.kr (N.T.N.T.); jhjung@ynu.ac.kr (J.J.H.); \\ Tel.: +82-053-8103513 (N.T.N.T.); +82-053-8103513 (J.J.H.)
}

Received: 3 May 2019; Accepted: 31 May 2019; Published: 8 June 2019

\begin{abstract}
Czochralski crystal growth has become a popular technique to produce pure single crystals. Many methods have also been developed to optimize this process. In this study, a charge-coupled device camera was used to record the crystal growth progress from beginning to end. The device outputs images which were then used to create a classifier using the Haar-cascade and AdaBoost algorithms. After the classifier was generated, artificial intelligence (AI) was used to recognize the images obtained from good dipping and calculate the duration of this operating. This optimization approach improved a Czochralski which can detect a good dipping step automatically and measure the duration with high accuracy. Using this development, the labor cost of the Czochralski system can be reduced by changing the contribution of human specialists' mission.
\end{abstract}

Keywords: Czochralski process; Haar-cascade algorithm; image processing; optimization

\section{Introduction}

Since the development of coal-operated steam machine (Savery) in 1710, gasoline machines in 1880, and the first "new machine magneto-electrique produisant de courant continu" was brought to public by Gramme in 1871, the demand for fossil fuels, gasoline, and electricity has constantly risen to satisfy worldwide demand [1]. People in the past relied heavily on nonrenewable energy sources such as wood, coal, and gasoline, but are now switching to renewable energy sources such as hydro, wind, and solar energy. Approximately $94 \%$ of the world energy demand in 1900 was met by wood and coal, which still contributed 73\% of the world's energy in 1973 and 38\% in 2000 [1]. Biofuels supplied 12\% of world energy in 2000 and only $8 \%$ in 2015 [1]. In 2015, hydro, wind, and solar power contributed $16 \%, 3.5 \%$, and $1 \%$ of the world energy, respectively; but are predicted to rise by a factor of 2.5 by 2020 [1]. This study developed an upgrading way for fabricating single crystal silicon ingots, which is a basic material of the solar module to create solar cells to harness solar energy. Solar cells are typically composed of synthetic single Si crystals or bulk single crystal [2]. Many methods are used for the production of bulk crystal single crystals-referred to as "crystal growth"-including solution, vapor, and melt growths. In the melt growth field, techniques such as crystal pulling, directional solidification, and the floating zone Verneuil know-how are used. The Czochralski (CZ) technique-a crystal pulling process-is the most important approach used to fabricate free silicon crystals with diameters as large as $300 \mathrm{~mm}$, weighing as much as $300 \mathrm{~kg}$ (large scale and diameter single crystal) [2,3]. The shape and quality of the crystal are influenced by the heating power, pulling rate, rotation rate and thermal gradient [2]. This study aims to minimize the operating cost of monocrystalline silicon growth and to produce high-quality products by applying computer vision, image processing algorithms, and machine learning to develop an automatic $\mathrm{CZ}$ system, which is typically performed by human specialists. 


\section{Materials and Methods}

\subsection{Related Work}

The $\mathrm{CZ}$ technique begins when the feed material is placed into the cylindrically-shaped graphite-quartz crucible and melted using a carbon heater. After material is molten, a few-millimeter-sized silicon seed is dipped from the top into the free melt surface and the melt meniscus is formed. After the remelting of a small portion of the dipped seed, the seed is slowly withdrawn from the melt with rotational force and the melt crystallizes at the interface of the seed, forming a new crystal portion. The crystal growth process was captured by a camera located at the top of the chamber, as shown in Figure 1. Since 1917, many designs have been developed to optimize the CZ, such as increasing the growth velocity to control the shape of the resulting bulk crystal [3], and dislocation-free growth by Teal and Little [4]. Then in 1975, Digges et al. introduced an optical sensing of the bright ring for automatic diameter control that indirectly measures the crystal diameter with rapid cooling methods [5]. In the 1990, Itsumi et al. applied octahedron structures for $\mathrm{SiO}_{2}$ using transmission electron microscopy and energy-dispersive $x$-ray spectroscopy [6]. An approach using 3D model from a transverse magnetic field in $\mathrm{CZ}$ furnace for predicting value of silicon growth rate with a pulling rate, rotation rate, and thermal gradients was also developed [7]. Moreover, to meet the demands of the next generation of the industry, computer science, biology, and chemistry must be integrated to develop unique solutions for complex problems. Many studies have been published regarding the application of computer science to this procedure. For example, Winkler et al. used a mathematic algorithm to calculate the crystal diameter and introduce the automatic CZ process with a nonlinear-state smart sensor, or soft sensor, or virtual sensor for controlling the quantities in the Czochralski crystal growth [8,9]. Jing et al. used a 3D-simulation method for investigating the relationship among the melting temperature at the melt or crystal surface, the rotation rate and efficiency of the final crystal product [10], and Kim et al. used an auto-seeding system and image processing technology to optimize the manufacturing of sapphire crystal growth via the Kyropoulos (KY) method [11]. Alternatively, a Malvern PVS system (Citrix Provisioning Services) was used to create 85 shape descriptors to analyze the crystal size and shape with the temperature and turbidity rate per minute [12,13]. Moreover, the aspect of crystal shape and quality also depends on the thermal gradient $\mathrm{G}$ managed by the thermal insulation and presence of the afterheater [14].

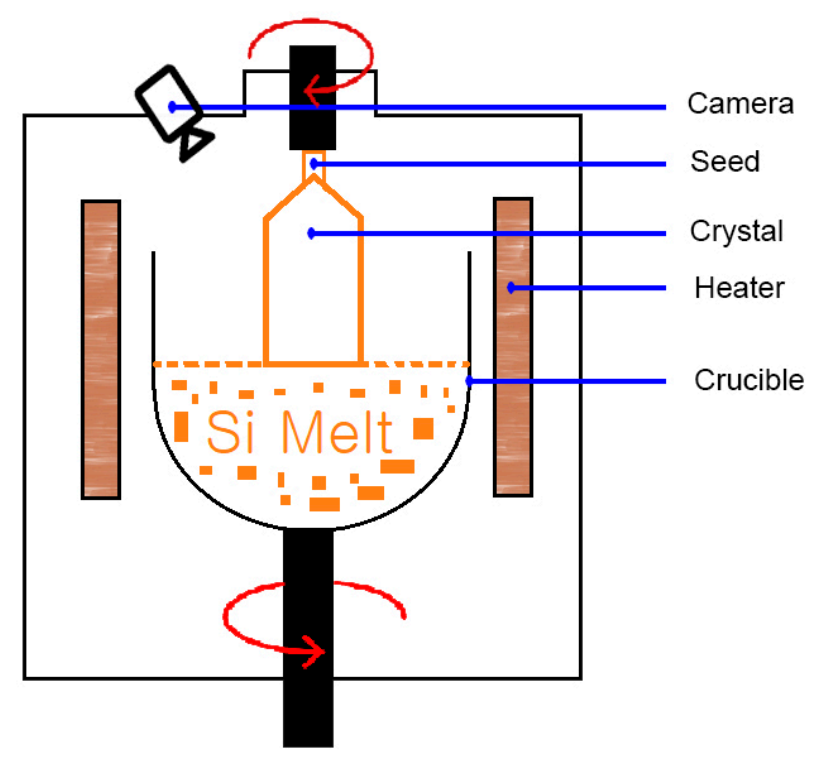

Figure 1. Czochralski crystal growth process schematic. 
The crystal growth progress is typically performed at $1600^{\circ} \mathrm{C}$ which is controlled by an external cryomagnet and thermometer installed in the chamber. Moreover, in order to detect the shape and quality of the crystal, there are strongly monitoring activities required: Images of the surface melt or crystal, the luminance of images, calculating of rotating speed, and thermal gradient. These actions are too hard to complete by human hands without the assistance of a computer [15]. Therefore, [7] a charge-coupled device (CCD) camera was installed to the device to handle some situations happening inside the $\mathrm{CZ}$ chamber. Additionally, the $\mathrm{CZ}$ system requires a very long time to finish a single crystal ingot [3], which also requires a specialist to follow and monitor the equipment. Factories that use CZ for ingot production have started to employ a combination of human labor and electronic assisting tools. Herein, an improving solution was applied to reduce human stress, reduce labor costs, and maintain the quality and speed of the $\mathrm{CZ}$ process. This solution involved computer technologies, such as computer vision, image processing, and machine learning for optimizing CZ.

\subsection{Applying Haar Cascade in Dipping Step}

\subsubsection{Applying Image Processing and Computer Data Analysis}

First, the chamber was recorded by the installed camera, as shown schematically in Figure 1. This camera outputted many pictures which were then applied a thresholding using preprocessing image algorithms for data reduction. Later, these data were labeled with different names to detect a good dipping object and its period which is a duration time when a good dipping object appears. This data monitoring helps a system to optimize the $C Z$ to be less reliant on human specialists [16]. The camera used in this study was a Sony IMX035 CMOS camera with a rolling shutter operated at 120 frames per second (fps) and pixel size at $3 \mu \mathrm{m}$. This CCD device was also used in the studies reported by Jin [17] and Jun et al. [18]. The capture period was during the early pulling stage when the seed was dipped into the melt, following which the machine used a twists and pull-up force to create a neck (necking stage as shown in Figure 2).

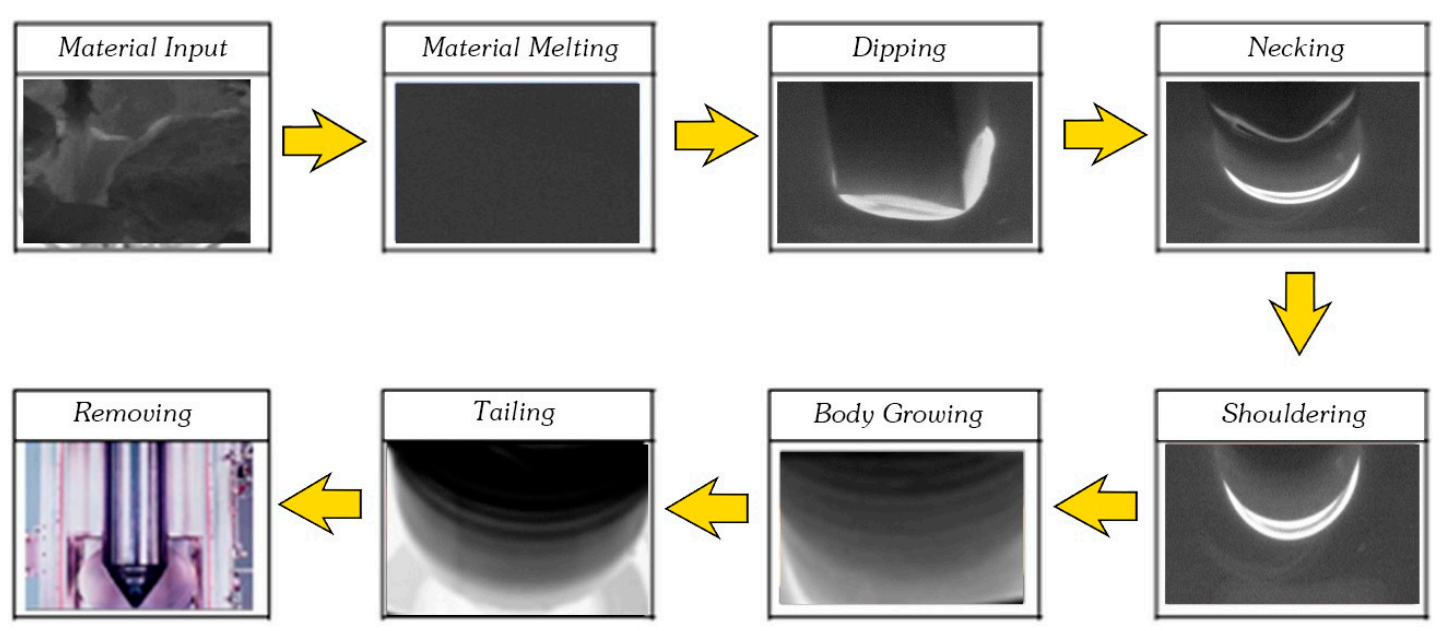

Figure 2. The Czochralski process from beginning to end.

During this stage, the bright gray solid-liquid interface in the image was the main focus and was captured for analysis by image preprocessing, image classification, and training steps (Figure 3). This section can be divided into the preprocessing steps including region extraction, color channel switching, noise reduction, and normal binary threshold, and processing steps where the shape of the considered objects were extracted, classified, and used to train the artificial intelligence (AI). 


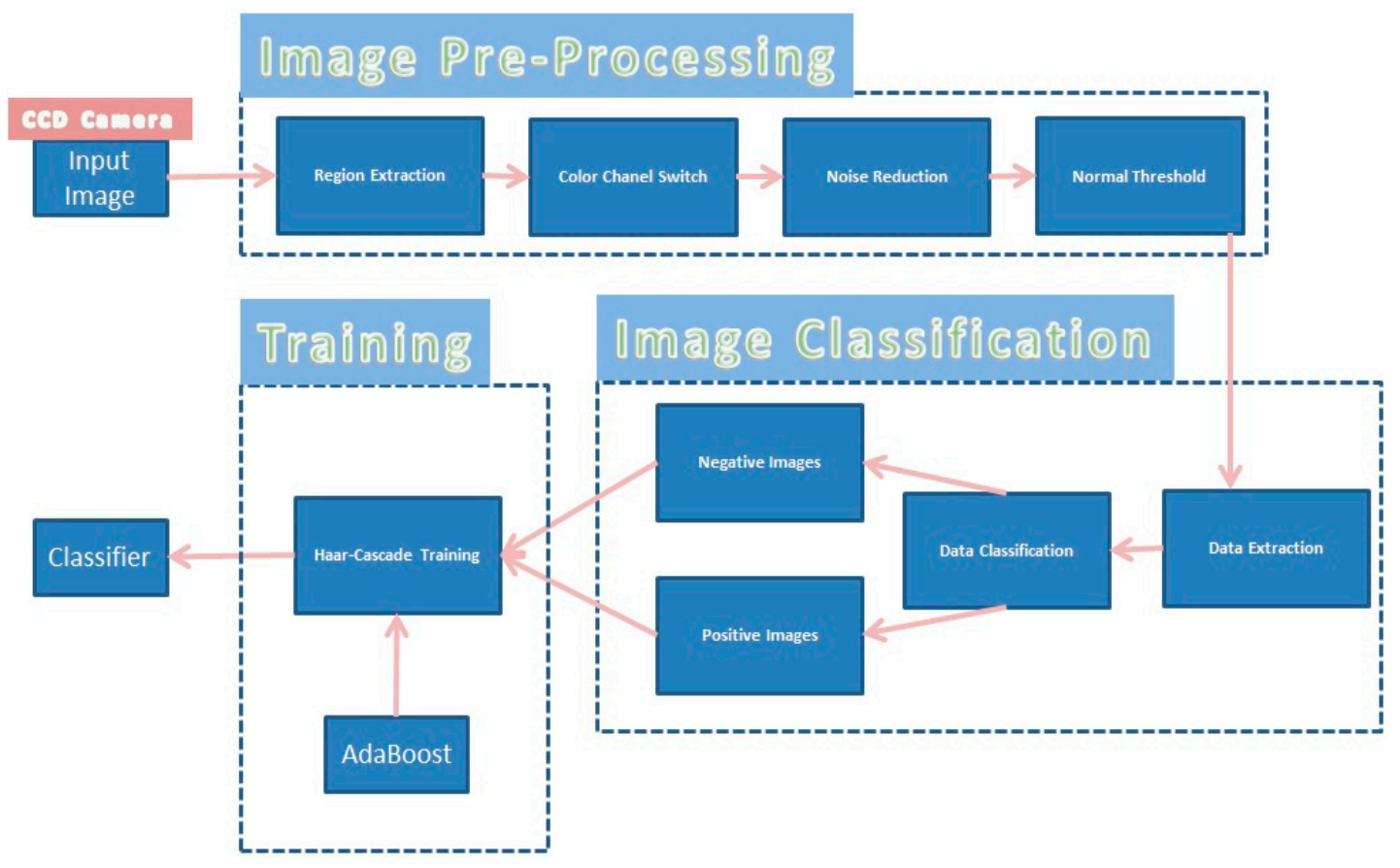

Figure 3. Optimizing the Czochralski process system structure.

\subsubsection{Detecting the Shape Using an Algorithm}

The outputted images were collected from the CCD, and the database contained many images. Each input was $1600 \times 1200$ pixels, but the focus area was $480 \times 380$ pixels, as shown in Figure 4 . Therefore, region extraction helped the computer calculate the information faster. The extracted region was $50 \times 50$ pixels, where the focus was solely on the area containing the object, as shown in Figure $4 \mathrm{~b}$. Later, the image was converted into the grayscale channel because the color channel (RGB, HIS, or LAB) made the objects more complex without any usefulness in detecting the shape of object and the threshold and edge detecting algorithms performed better on the grayscale channel (Figure 4c). After switching to the grayscale channel, a Gaussian filter (Gaussian blur) as Equation (1) was applied for each entry (pixel) by replacement with the median of neighboring entries (Figure 4d).

$$
G(x, y)=\frac{1}{2 \pi \sigma^{2}} \mathrm{e}^{-\left(x^{2}+y^{2}\right) /\left(2 \sigma^{2}\right)},
$$

where $x$ is the distance from the origin in the horizontal axis, $y$ is the distance from the origin in the vertical axis, and $\sigma$ is the standard deviation of the Gaussian distribution.

This step was performed to remove image noise created from imperceptible specks. During the next step, the thresholding algorithm, as Equation (2), was applied to the image to partition it into multiple segments for simplifying and analyzing the associated data (Figure 4e). The final images from this section were used for machine learning purposes.

$$
g(x, y)=\left\{\begin{array}{rr}
1 & \text { if } f(x, y) \geq T \\
0 & \text { otherwise }
\end{array}\right.
$$

where $g(x, y)$ is a thresholded version of $f(x, y)$ at $T$ (calculated based on histogram as Equation (3)).

$$
T=(p 1+p 2) / 2,
$$

where $p 1$ and $p 2$ are the gray value of the peaks of the histogram. 
Original Image

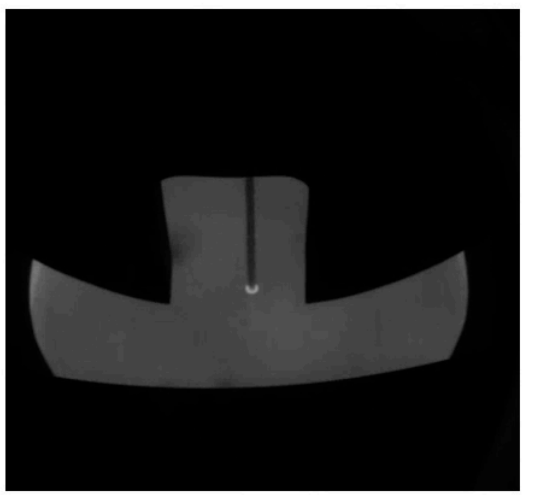

(a)
Focus

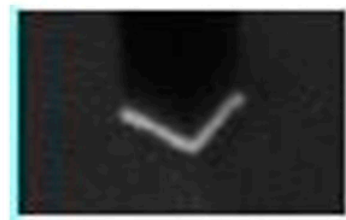

(b)

Blur

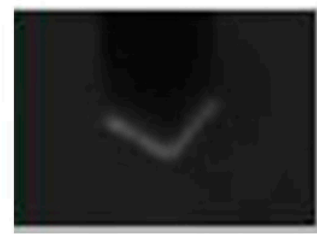

(d)
Gray Scale

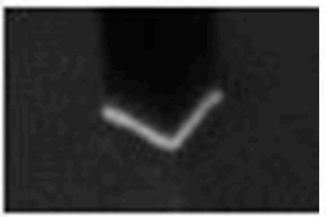

(c)

Threshold

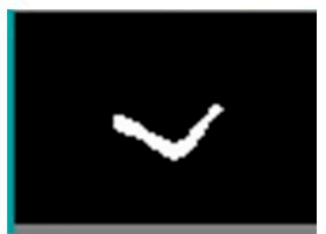

(e)

Figure 4. Images from the preprocessing step. (a) The original image; (b) images in the focusing step; (c) image in the grayscale channel; (d) image with the blur algorithm applied; (e) image with the binary threshold algorithm applied.

\subsubsection{Classifying the Shape Obtained}

After obtaining the final images from Section 3, a specialist classified whether the images represented "good data" which belong to a good dipping process (good images). The good images were labelled as "good dipping." The rest of the images were labeled as "negative." Later, the machine learning algorithm employing the Haar cascade was used as a classifier tool for object recognition. To complete the learning step, the good dipping images were grouped in a folder named "positive" and the negative images were grouped in a folder named "negative" (Figure 5). The positive image contained the need-to-find object and the negative image did not contain the need-to-find object. The need-to-find object (Figure 6) was defined as the good dipping image.
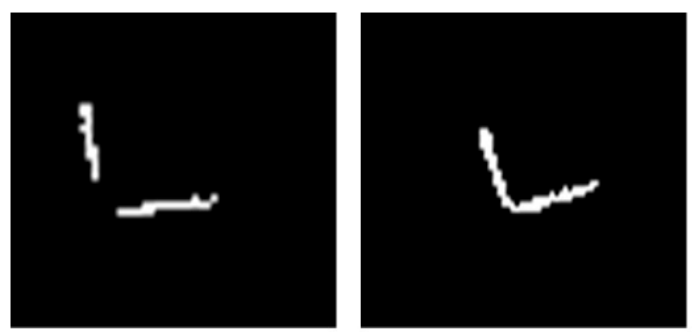

(a)
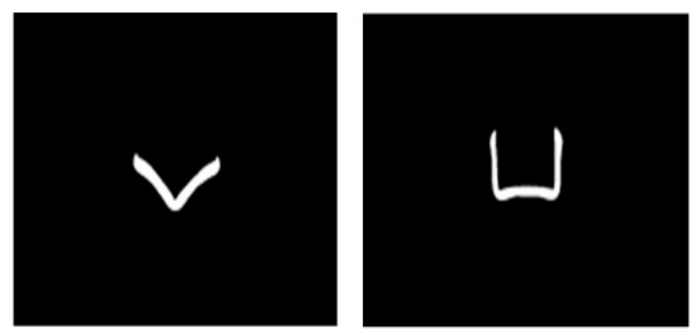

(b)

Figure 5. Negative and positive images: (a) Representative negative images; (b) representative positive images. 

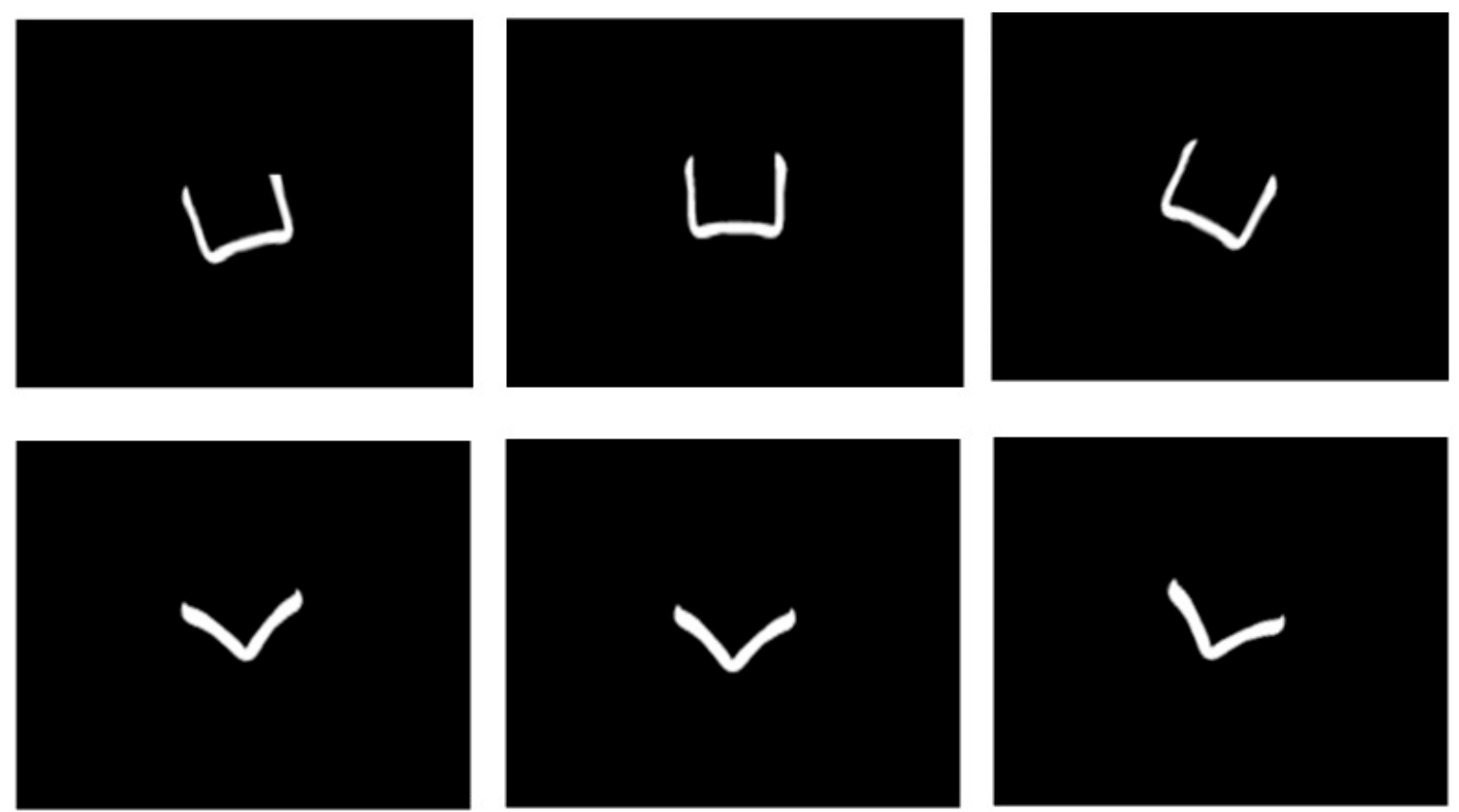

Figure 6. Examples of need-to-find objects (good dipping images).

\subsubsection{Training the Machine to Recognize the Need-to-Find Object}

After the classification step, the two sets of images were used for the training step. In this step, the AdaBoost learning algorithm was used to increase the classification performance of the Haar-cascade classifier training $[19,20]$. AdaBoost contains a large number of examples that had a strong effect on the generalization performance of a strong classifier's training error. This caused a small number of the images containing the need-to-find object to be misclassified. The AdaBoost algorithm simultaneously runs many learning steps. The purpose of the learning method was to construct a classifier for the recognition of good dipping images and ideal durations in the next step. This learning has many states that must be decided by the user. For each state, the computer creates a first classifier from the positive images and tests it on the negative images for evaluation and builds a second classifier featuring higher detection rates. The second classifier is subsequently used in the next states. This process ends when the last state is completed. The cascade stages are run by training the classifier tool using the AdaBoost algorithm and compiling with the threshold algorithm to minimize the error rate.

\subsubsection{Applying the Cascade Classifier for Detecting Good Dipping}

After running the cascade, a classifier file XML was generated containing the results of the training step. With this classifier, a software was developed to monitor the $\mathrm{CZ}$ process by viewing the pictures captured by the CCD and detecting images with ROI (regions of interest) containing a good dipping object and giving a warming signal. Moreover, the duration was logged from time A, when the first good dipping object was found, to time B when no good dipping object was present. When the duration was sufficient, the necking step was started automatically. The rest of the CZ system was normally operated by the machine. The two sets of images were input into the cascade-training process with the data listed in Table 1. When the first good dipping object was recognized, the system time (time A) was recorded. When the last good dipping object was found, the system time (time B) was counted. The duration (good dipping time) was defined as the difference between time A and B. This time was used for the comparison with the actual duration of the CZ process. The software is developed to detect a good dipping object, the duration time. When the duration time is enough, the software outputs a signal to move from dipping process to necking process in CZ, as the following Figure 7. 
Table 1. Haar-cascade training technical data.

\begin{tabular}{cccccc}
\hline Name & Data & Name & Data & Name & Data \\
\hline Number of States: & 20 & Boost Type: & GAB & Maximal Weak Tree: & 100 \\
\hline Number of Threads: & 7 & Minimal Hit Rate: & 0.995 & Number of Positives: & 1150 \\
\hline $\begin{array}{c}\text { Acceptance Ratio } \\
\text { Break Value: }\end{array}$ & -1 & $\begin{array}{c}\text { Maximal False } \\
\text { Alarm Rate: }\end{array}$ & 0.5 & $\begin{array}{c}\text { Number of } \\
\text { Negatives: }\end{array}$ & 8850 \\
\hline Feature Type: & Haar & Weight Trim Rate: & 0.95 & - & - \\
\hline Haar Feature Type: & Basic & $\begin{array}{c}\text { Maximal Depth } \\
\text { Weak Tree: }\end{array}$ & 1 & - & - \\
\hline
\end{tabular}

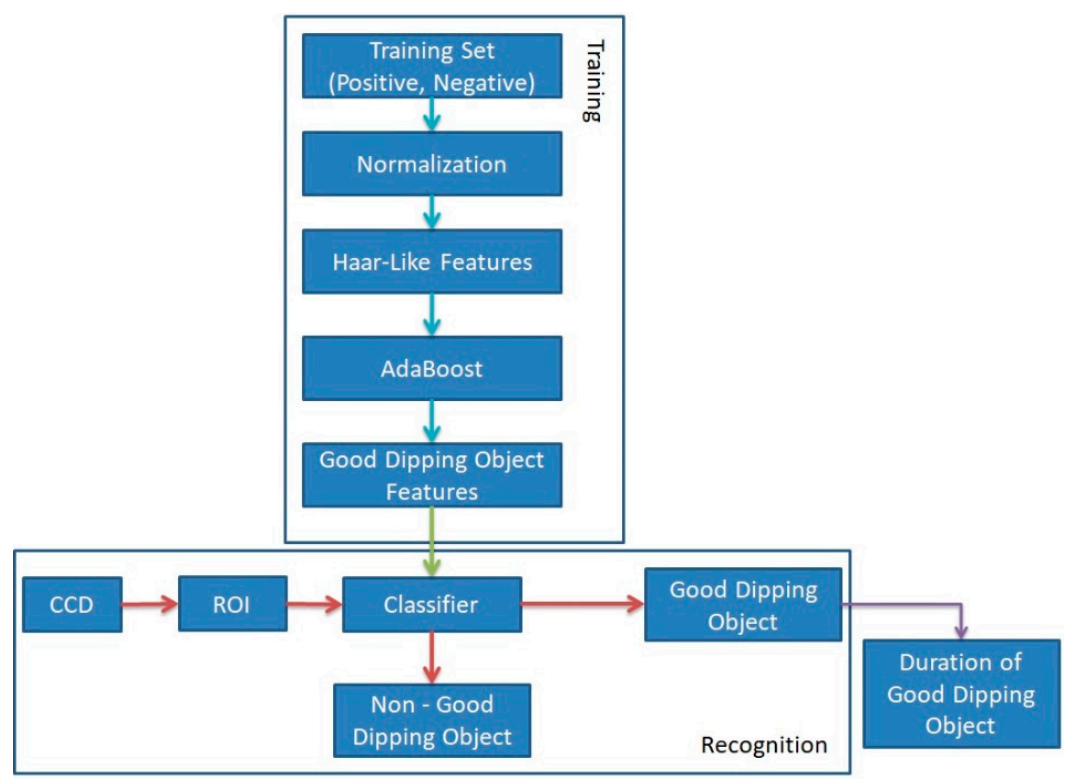

Figure 7. Applying Haar-cascade classifiers diagram.

\section{Experiment Results}

The CZ process begins with the material input step and ends with the removal (Figure 2). The most important thing is the dipping system, where the melting temperature and duration are extremely important. By applying this optimization method, good dipping images were detected with a high accuracy rate and the duration was calculated with very small errors. The first dataset recorded by CCD contained over 2 million images of $660 \times 550$ pixels. These images were preprocessed using several algorithms, including grayscale channel switching, threshold at specific value application, and median blur processing. Afterwards, the images were classified into two folders: Positive (approximately 11,000 ) and negative (approximately 90,000). These 90,000 images were obtained from different steps of $\mathrm{CZ}$, such as material input and melting.

After completing machine learning, the classifier file was used for detecting good dipping images. To draw a conclusion, five cases were created from recording different complete $\mathrm{CZ}$ operations (from material input to removal) with the same condition. In each case, the whole manufacturing was recorded by CCD as series of images. After five collections of images were obtained, the optimizing CZ was tested on each image on the computer. As shown in Table 2, in all five cases, the performance of the improved CZ (ICZ) was as efficient as the normal CZ controlled by a human specialist. The times of dipping and melting temperature between the two processes were slightly different. Under the $\mathrm{CCD}$, the object with a higher temperature appears brighter. Using the computer vision technique, the dipping period, which requires a specific range of melting temperature, and duration, can be controlled using a computer without human input. The speed of the cascade detector training depends on the size of the input database needed for each training stage. Therefore, the combination of the 
AdaBoost and Haar-cascade classifier algorithms was used to create a classifier to automatically detect good dipping objects and calculate the duration.

Table 2. Differences between the optimized CZ (ICZ) and the normal CZ dipping step.

\begin{tabular}{|c|c|c|c|c|c|}
\hline Case & $\begin{array}{l}\text { Process's } \\
\text { Name }\end{array}$ & $\begin{array}{l}\text { Good Dipping } \\
\text { Images }\end{array}$ & $\begin{array}{l}\text { False Detect } \\
\text { Images }\end{array}$ & $\begin{array}{c}\text { Accuracy Rate } \\
(\%)\end{array}$ & $\begin{array}{l}\text { Duration Time } \\
\text { Difference (s) }\end{array}$ \\
\hline \multirow{2}{*}{ Case 1} & ICZ & 11455 & 45 & 99.60869565 & 4.578 \\
\hline & Normal CZ & 11500 & & & \\
\hline \multirow{2}{*}{ Case 2} & ICZ & 11516 & 34 & 99.70562771 & 4.289 \\
\hline & Normal CZ & 11550 & & & \\
\hline \multirow{2}{*}{ Case 3} & ICZ & 11473 & 67 & 99.41941075 & 5.184 \\
\hline & Normal CZ & 11540 & & & \\
\hline \multirow{2}{*}{ Case 4} & ICZ & 11329 & 101 & 99.11636045 & 8.937 \\
\hline & Normal CZ & 11430 & & & \\
\hline \multirow{2}{*}{ Case 5} & ICZ & 11468 & 52 & 99.54861111 & 4.819 \\
\hline & Normal CZ & 11520 & & & \\
\hline
\end{tabular}

\section{Discussion}

In Table 2 the improving CZ (ICZ) refers the process that used computer vision detection and the normal CZ refers to the specialist-operated normal CZ (NCZ). The good dipping images in the ICZ were counted and obtained from the total number of images that the computer recognized. The good dipping images of NCZ were obtained from the actual images recorded from the actual CZ process. The false detection images are those containing non-good dipping object which were detected as a good dipping object or good dipping objects that were not detected. The accuracy rate is the percentage difference between the good dipping images recognized in the ICZ and NCZ processes as Figure 8 . The duration time difference is the difference between the good dipping time of ICZ and the actual time of the dipping step in the NCZ process as Figure 9. These results from dipping step among ICZ and CZ had a small difference that indicated the next necking step's start run with a slightly difference. With the same operating condition, the quality of the crystals obtained from ICZ was predicted as same as NCZ. Moreover, the efficiency of the ICZ process was quite high (>99.1\%). In case 4 , there was a slight decrease in accuracy rate and the small increase in duration time difference due to the images' illumination which affects the merging area of Haar-cascade algorithm [21].

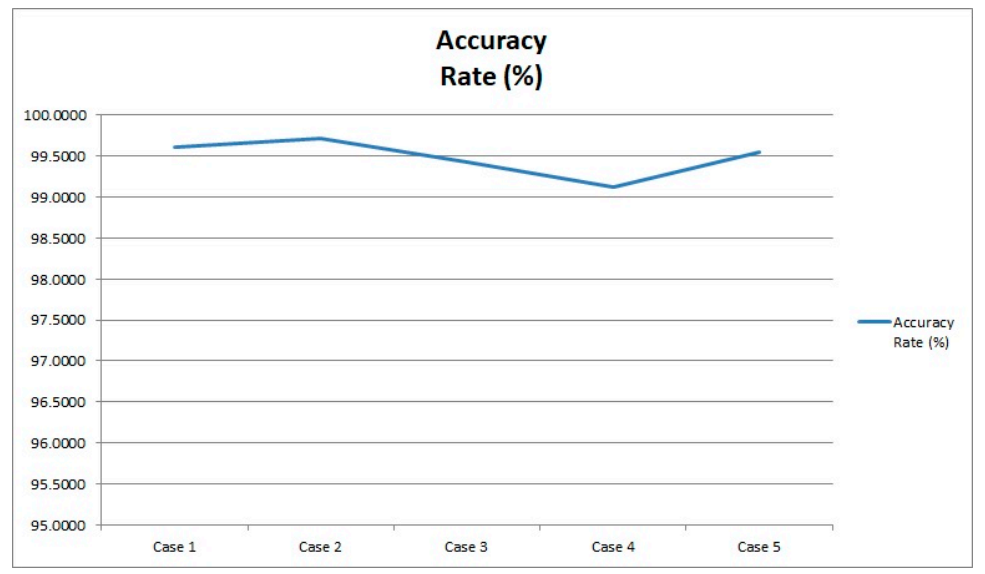

Figure 8. Detection accuracy rate in the five cases. 


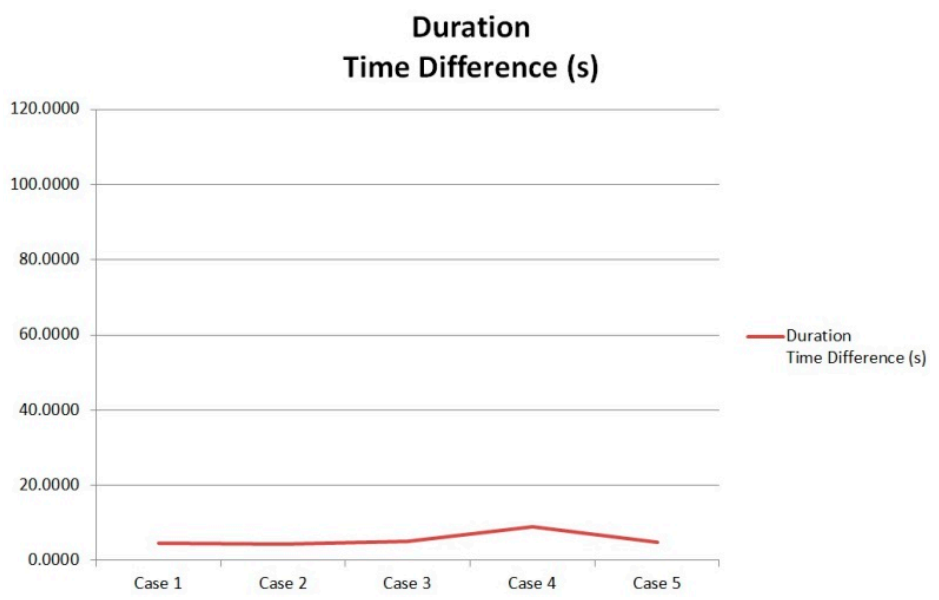

Figure 9. Duration differences in the five cases.

Haar cascade uses an integral image which calculates adjacent rectangular regions at a specific location in an object image, and then this algorithm sums up the pixel intensities in each region and calculates the difference between these sums to make a weak classifier with a fast speed. Later, this algorithm constructs a "strong" classifier as a linear combination of weighted simple "weak" classifiers. Moreover, the shape of good dipping object is identical and able to be detected with some image calculating. With the help of Haar cascade, the good dipping objects are detected and signaled back to the system about its appearance. With the newly developed ICZ, the quality of the final product (single pure crystal Si) was maintained without the need for human intervention. Therefore, this technique has great potential for reducing labor costs.

\section{Conclusion}

It is important to know the ideal time for switching from dipping to necking in the $\mathrm{CZ}$ system, which is usually determined by a human specialist. However, for the next generation of the industry, manufacturing will require increasing automation, depending less on human intervention. Therefore, the $\mathrm{CZ}$ operation was optimized using image processing and machine learning to adapt solar cell component manufacturing to the developing trends in the industry. This optimization required many CCD images from the normal CZ progress. Several preprocessing algorithms were applied to the obtained images for noise reduction. Subsequently, these images were labeled and trained using two algorithms, AdaBoost and Haar cascade, to generate a classifier. This classifier was then used to recognize good dipping objects and its duration. With these two types of data, the CZ system was programmed to automatically switch from dipping to necking. The application of this optimization resulted in a small difference compared to the standard CZ design. The developed approach will reduce the need for a specialist, which will reduce labor costs. In the future, a deep-learning algorithm which combines the images, melting temperature data, and object sizes will be developed to increase the accuracy of the object recognition method.

Author Contributions: Conceptualization, J.J.H. and N.T.N.T.; Methodology, J.J.H. and L.T.H.P.; Software, L.T.H.P.; Validation, J.J.H. and N.T.N.T.; Formal Analysis, J.J.H. and L.T.H.P.; Investigation, L.T.H.P. and H.J.; Resources, STech Co., Ltd and Process System Optimized Lab.; Data Curation, Process System Optimized Lab; Writing-Original Draft Preparation, L.T.H.P.; Writing-Review \& Editing, J.J.H. and L.T.H.P.; Visualization, J.J.H., L.T.H.P. and H.J.; Supervision, J.J.H. and N.T.N.T.; Project Administration, J.J.H..

Funding: This work was supported by the Korea Institute of Energy Technology Evaluation and Planning (KETEP) and Ministry of Trade, Industry and Energy (MOTIE) of Republic of Korea (No. 20173030018990). The image data used in this work are sponsored by the Process System Optimized Lab from Yeungnam University and S-Tech Company Limited ( S-Tech Co., Ltd.) from Korea (No. 20174030201760).

Conflicts of Interest: The authors declare no conflict of interest. 


\section{References}

1. Smil, V. Energy and Civilization: A History; Chapter 5; The MIT Press: Cambridge, MA, USA, 2017; pp. $237-295$.

2. Jochen, F.; Ammon, W.; Muller, G. Czochralski Growth of Silicon Crystals. In Handbook of Crystal Growth, 2nd ed.; Rudolph, P., Ed.; Elsevier: London, UK, 2015; pp. 45-104.

3. Czochraski, J. A new method for the measurement of the crystallization rate of metals. Z. Phys. Chem. 1918, 92, 219-221.

4. Teal, G.K.; Little, J.B. Growth of germanium single crystals. Phys. Rev. 1950, 78, 647.

5. Digges, T.G.; Hopkins, R.H.; Seidensticker, R.G. The basis of automatic diameter control utilizing "bright ring" meniscus reflections. J. Cryst. Growth 1975, 29, 326-328. [CrossRef]

6. Itsumi, M.; Akiya, H.; Ueki, T. The composition of octahedron structures that act as an origin of defects in thermal $\mathrm{SiO}_{2}$ on Czochralski silicon. J. Appl. Phys. 1995, 78, 5984. [CrossRef]

7. Kakimto, K. Development of Crystal Growth Technique of Silicon by Czochralski Method. Acta Phys. Pol. A 2013, 124, 227-230. [CrossRef]

8. Winkler, J.; Robenack, K. Observer based determination of the crystal diameter in Czochralski crystal growth utilizing algorithmic differentiation. In Proceedings of the 2012 9th International Multi-Conference on Systems, Signal and Devices (SSD), Chemnitz, Germany, 20-23 March 2012; pp. 1-6.

9. Winkler, J.; Neubert, M.; Rudolph, J. A review of the automation of the Czochralski crystal growth process. Acta Phys. Pol. 2013, 124, 181-192. [CrossRef]

10. Jing, C.J.; Imaishi, N.; Yasuhiro, S.; Miyazawa, Y. Three-dimensional numerical simulation of oxide melt flow in Czochralski configuration. J. Cryst. Growth 2016, 216, 372-388. [CrossRef]

11. Kim, C.M.; Kim, S.R.; Ahn, J.H. Development of Auto-seeding System Using Image Processing Technology in the Sapphire Crystal Growth Process via the Kyropoulos Mehod. Appl. Sci. 2017, 7, 371. [CrossRef]

12. Wang, X.Z.; de Anda, J.C.; Roberts, K.J. Real-Time Measurement of the Growth Rates of Individual Crystal Facets Using Imaging and Image Analysis: A Feasibility Study on Needle-shaped Crystal of L-Glutamic Acid. Chem. Eng. Res. Des. 2007, 85, 921-927. [CrossRef]

13. Wang, X.; Roberts, K.; Ma, C. Crystal Growth measurement using 2d and 3d imaging and the perspectives for shape control. Chem. Eng. Des. 2008, 63, 1173-1184. [CrossRef]

14. Mohammad, H.T.; Shirin, O.; Ebrahim, M. Influence of active afterheater on the fluid dynamics and heat transfer during Czochralski growth of oxide single crystals. CrystEngComm 2011, 13, 5088-5093.

15. Ding, J.; Liu, L. Real-time prediction of crystal/melt interface shape during Czochraski crystal growth. CrystEngComm 2018, 20, 6925-6931. [CrossRef]

16. Amit, K.; Jyotirmay, B.; Muralidhar, K. Thermal Modeling of Crystal Growth by Czochraski Method including Radius Control. J. Sci. Ind. Res. 2002, 61, 607-616.

17. Jin, T.; Shengdeng, W.; Jianwei, C. Research on Measure Method of Diameter of Partial Circle Based on CCD. Semicond. Technol. 2007, 32, 574-576.

18. Han, X.J.; Wang, X.M.; Zhao, Q. Image Processing for the use of crystal production monitor. Chin. J. Sens. Actuators 2006, 19, 346-348.

19. Viola, P.; Jones, M. Rapid Object Detector using a Boost Cascade of Simple Features, Rapid object detection using a boosted cascade of simple features. IEEE CVPR 2001, 1, 511-518.

20. Yoav, F.; Robert, E.S. A Short Introduction to Boosting. J. Jpn. Soc. Artif. Intell. 1999, 14, 771-780.

21. Soo, S. Seminar University of Tartu. Available online: http://ds.cs.ut.ee/Members/artjom85/2014dss-coursemedia/Object\%20detection\%20using\%20Haar-final.pdf (accessed on 14 May 2019).

(C) 2019 by the authors. Licensee MDPI, Basel, Switzerland. This article is an open access article distributed under the terms and conditions of the Creative Commons Attribution (CC BY) license (http://creativecommons.org/licenses/by/4.0/). 